\title{
Scholarly Sharing via Twitter: \#icanhazpdf Requests for Health Sciences Literature ${ }^{1}$
}

\author{
Michelle Swab and Kristen Romme
}

\begin{abstract}
Introduction: Although requesting access to journal articles and books via colleagues and authors is a longestablished academic practice, websites and social media platforms have broadened the scope and visibility of academic literature sharing among researchers. On Twitter, the \#icanhazpdf hashtag has emerged as a way for researchers to request and obtain journal articles quickly and efficiently. This study analyzes use of the \#icanhazpdf hashtag as a means of obtaining health sciences literature. Methods: RowFeeder software was used to monitor and aggregate \#icanhazpdf requests between 1 February and 30 April 2015. This software records data such as Twitter handle, tweet content, tweeter location, date, and time. Tweets were hand-coded for the journal subject area, the requestor's geographic location, and the requestor's occupational sector. Results: There were 302 requests for health sciences literature during the study period. Many requests were made by users affiliated with a post-secondary academic institution $(45 \%, n=136)$. Very few requests were made by users located in Canada $(n=15)$. Conclusion: \#icanhazpdf requests for health sciences literature account for a relatively small proportion of peer-to-peer article sharing activities when compared with other online platforms. Nevertheless, this study provides evidence that some faculty and students are choosing social media over the library as a means of obtaining health sciences literature. Examining peer-to-peer article sharing practices can provide insights into patron behaviour and expectations.
\end{abstract}

\section{Introduction}

The proportion of biomedical literature available through Open Access (OA) publishing models is increasing. In a 2013 survey study, Kurata et al. [1] found that the proportion of medical research articles available OA doubled between 2006 and 2010. However, many articles remain inaccessible to those without personal or institutional subscriptions to scholarly journals. In this environment, alternative article distribution channels that facilitate peer-to-peer sharing have emerged.

Researchers have long been able to bypass libraries and journal publishers by requesting research articles directly from article authors or colleagues, a practice Salo [2] terms "academic samizdat". Online environments have enabled new forms of this practice, allowing researchers to request articles from large networks of people on a variety of websites, discussion forums, and social media platforms.

The overall extent of article sharing is difficult to determine due to shifting online landscapes; some dedicated article sharing websites such as journalfire.com and scientificcommons.org are now defunct. In the meantime, other websites and tools have taken their place. In a 2005 paper, Wren [3] estimated that over one-third of high impact journal articles published in 2003 could be found on non-journal websites indexed by Google. Other studies have examined sharing on specific platforms. Masters [4] analyzed peer-to-peer article sharing on a website popular with medical professionals, and documented 5464 full-text article pdf postings in response to requests during a sixmonth period in 2008 [4]. One of the most extensive studies to date was conducted by Cabanac, who examined full-text content available on the Library Genesis (LibGen) platform. LibGen hosted over 25 million documents at the time of the study; $36 \%$ of all articles assigned a digital object identifier (DOI) were found to be available on the platform, with $71 \%$ of the content originating from massive paper uploads and $29 \%$ originating from crowd-sourced sites [5].

The growth of online peer-to-peer article sharing has a variety of implications for libraries. Greenhill and Wiebrand [6] argue that peer-to-peer article sharing serves as a "hidden competitor" for libraries. Similarly, England and Jones [7] remark that peer-to-peer article sharing "represents a worrying trend toward disintermediation of the library and negatively impacts the library's perceived relevance and value among students and faculty" [7].

Given the potential impacts of article sharing for health sciences libraries, it is important to understand how and

Michelle Swab, MA MLIS ${ }^{2}$, Kristen Romme, MA MLIS, Health Sciences Library, Memorial University of Newfoundland, St. John's,

NL.

${ }^{1}$ This article has been peer-reviewed.

${ }^{2}$ Corresponding author (email: mswab@mun.ca) 
why medical literature is being shared online. This paper investigates article requests via one particular method, the \#icanhazpdf Twitter hashtag ${ }^{3}$. Specific research questions include:

- How often is \#icanhazpdf used to request health sciences literature?

- What types of health sciences literature are being requested?

- How do users identify requested literature (i.e., publisher URL, DOI, or citation information)?

- Where are requestors geographically located?

- What is the occupation or employment sector of \#icanhazpdf tweeters requesting health sciences literature?

- Do article requestors have access to library resources and services?

Copyright and licensing issues surrounding peer-to-peer article sharing practices such as \#icanhazpdf are complex and situational. In some instances, \#icanhazpdf users may be in violation of copyright legislation or publisher licensing agreements, but those who use the hashtag may argue that this practice typically falls under fair use or fair dealing exceptions. To add to the confusion, some publishers permit one-to-one, private article sharing among colleagues [8]. Given the many competing viewpoints, a detailed examination of the intricacies of copyright legislation and publisher licensing in relation to \#icanhazpdf is beyond the scope of this paper.

\section{How does \#icanhazpdf work?}

San Francisco-based cognitive scientist Andrea Kuszewski first conceived of the \#icanhazpdf hashtag in 2011 [9]. According to \#icanhazpdf protocol, article requestors compose a tweet containing article information, their personal email address, and the \#icanhazpdf hashtag (Figure 1). Other Twitter users fulfill requests by searching Twitter for the \#icanhazpdf hashtag, accessing requested articles through institutional or personal subscriptions, and then emailing the article to the requestor. Once the request has been fulfilled, the requestor deletes the tweet. This procedure maintains anonymity for the article provider as he or she may infringe copyright or be in violation of licensing agreements.

Fig. 1. Sample tweet.

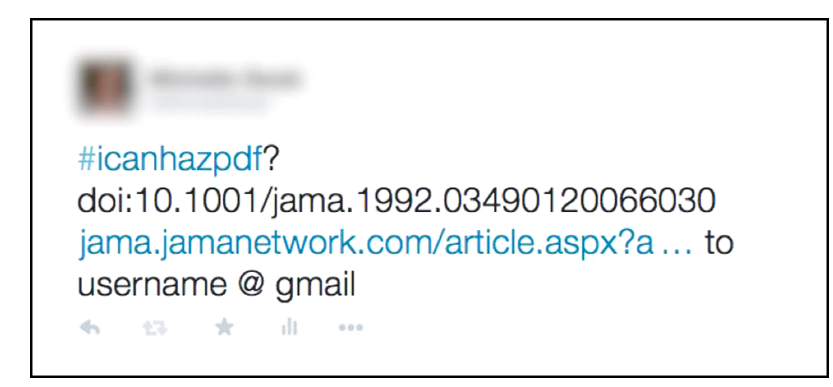

\footnotetext{
${ }^{3}$ \#icanhazpdf Twitter feed is updated continuously and is available at https://twitter.com/\#!/search?q=023icanhazpdf.
}

Liu [10] notes that the hashtag was originally intended for science journalists, who typically lack access to the online library resources available to researchers at large universities; however, her research has demonstrated that academics and students use \#icanhazpdf services more frequently than those in communication fields.

A 2015 paper by Gardner and Gardner [9] also provides a variety of insights into the use of \#icanhazpdf. Of particular importance, their study found that $62 \%$ of requests were for articles from life sciences and biomedical journals. This paper further examines this particular category of requests.

\section{Methods}

As \#icanhazpdf protocol dictates that tweets are deleted after the requested article is received, the social media monitoring service RowFeeder was used to capture and collect publicly available \#icanhazpdf tweets. RowFeeder, which is a subscription service, records the Twitter username, the content of the tweet, the tweet URL, the user location (if supplied), and the date and time of the tweet [11]. Memorial University's Ethics Officer advised that ethics approval was not required for this research as individual \#icanhazpdf users would not be identified.

RowFeeder recorded a total of 3962 tweets during the period 1 February to 30 April 2015. Many of the captured tweets were automated retweets by the Twitter bots hecanhazpdf and i_can_haz_PDF. Such tweets were excluded from the dataset, as were retweets by individuals. The remaining 1456 unique tweets were divided into two sets (Figure 2). Each author manually reviewed one set of tweets, coding each tweet with the following demographic information and request details: requestor's geographic region (if known); type of tweet (request or comment); type of material requested (article, book, etc.); how the requested item was described (citation, DOI, link to publisher, etc.); and subject of the requested item (health or non-health). Requests for health sciences literature were further coded with Scopus subject categories at the journal level. For example, a request for an article from Nature Reviews Microbiology was coded with the subject areas "Immunology and Microbiology" and "Medicine," which are the subject categories that Scopus assigns to the journal. If questions or uncertainty arose during the process of coding, both authors reviewed the tweet in question and came to a consensus.

\section{Results}

In total, there were 302 requests for health sciences literature using the \#icanhazpdf Twitter hashtag between 1 February and 30 April 2015. Nearly all of the requests $(99 \%, n=300)$ were for journal articles. Of the two nonarticle requests, one was for a book and the other for grey literature.

\section{Characteristics of requested articles}

The 300 health sciences articles requested were from 232 different journals. Journals could be classified in more than one category; see Table 1 for the full list of subject areas for which articles were requested. 
Fig. 2. Tweet inclusion flow chart.

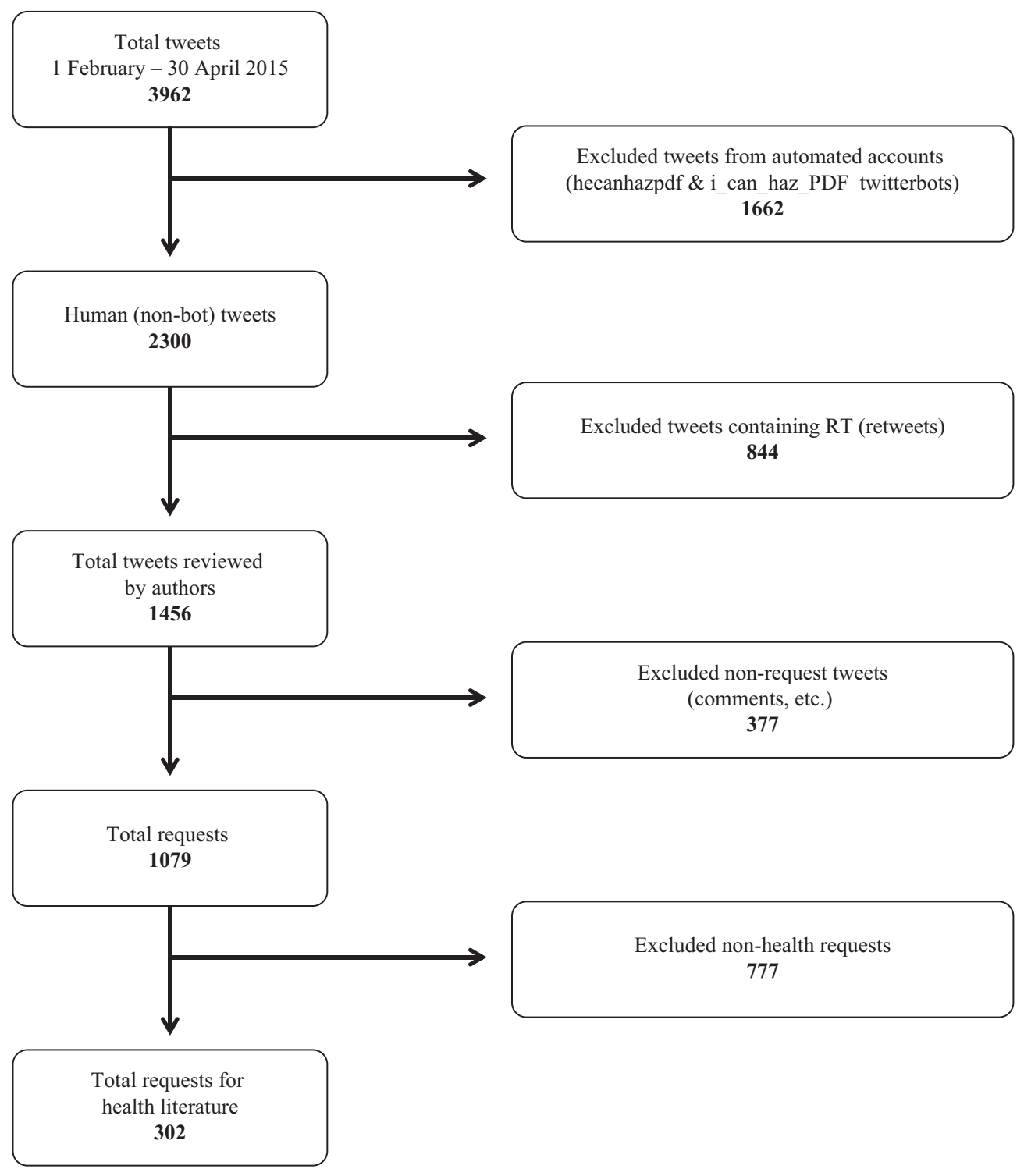

Table 1. Scopus subject category for unique journal titles.

\begin{tabular}{lc}
\hline Broad journal subject category & Number of journals* \\
\hline Medicine & 196 \\
Nursing & 14 \\
Pharmacology & 18 \\
Immunology and microbiology & 22 \\
Health professions & 5 \\
Clinical psychology & 2 \\
Dentistry & 1 \\
Not indexed in Scopus & 7 \\
\hline
\end{tabular}

*Journal titles may be classified in more than one category.

More than half of requestors $(50.7 \%, n=152)$ linked to the publisher's website as a means of identifying the article being requested (Figure 3). Linking to the PubMed record was the second most popular means of identification.

\section{Characteristics of requestors}

As shown in Table 2, our findings are consistent with Gardner and Gardner's [9] assertion that "\#icanhazPDF is overwhelmingly an Anglophone phenomenon". The greatest proportion of requests came from the United Kingdom and Ireland (29.1\%), followed by the United States $(26.5 \%)$. There were comparatively few \#icanhazpdf requests for health literature from Canada: only 15 requests in the 3 months of the study.

Many requests $(45 \%, n=136)$ came from Twitter users who were in some way affiliated with a post-secondary academic institution, according to the information provided in their Twitter profile. These requestors included faculty, academic researchers, students, and librarians. The number of requestors with academic affiliation may in fact be even greater, as academic affiliation could not be determined for over $38 \%(n=117)$ of requestors (Table 3$)$. 
Fig. 3. Description of requested materials $(n=302)$.

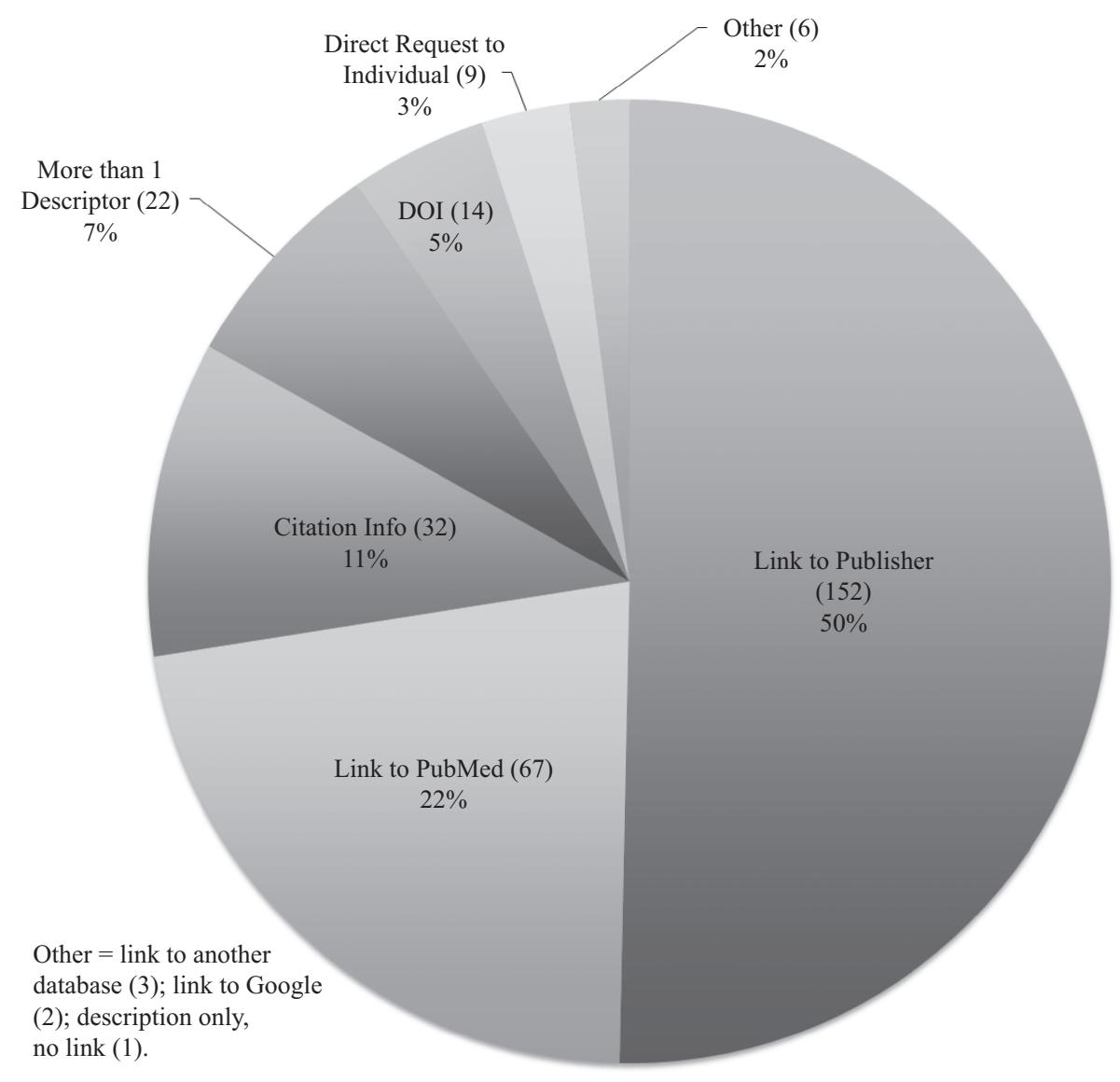

\section{Discussion}

The overall number of requests for health sciences literature on Twitter using the \#icanhazpdf hashtag during the three-month study period was low $(n=302)$ in comparison with requests for articles on a website for health professionals during a six-month period in 2008 as reported by Masters $(n=6587)$ [4]. Few \#icanhazpdf requests for health sciences literature during the study period originated from Canadian Twitter users $(n=15)$. The low rate of \#icanhazpdf use in Canada may be related to the low rate of Twitter adoption among Canadian health professionals

Table 2. Requestor's geographic region.

\begin{tabular}{lcc}
\hline & Number of tweets & Percent total \\
\hline United Kingdom and Ireland & 88 & $29.1 \%$ \\
United States & 80 & $26.5 \%$ \\
Rest of Europe & 58 & $19.2 \%$ \\
Canada & 15 & $5.0 \%$ \\
Australia and New Zealand & 11 & $3.6 \%$ \\
Asia & 3 & $1.0 \%$ \\
Mexico, Central America, & 2 & $0.7 \%$ \\
$\quad$ South America & & \\
Africa & 1 & $0.3 \%$ \\
Unknown & 44 & $14.6 \%$ \\
Total & 302 & $100.0 \%$ \\
\hline
\end{tabular}

and researchers. Only $9.2 \%$ of respondents in a November 2013 survey of Canadian Medical Association physicians reported using Twitter for professional purposes [12], and only $5 \%$ of faculty respondents reported Twitter use in a recent survey conducted at the University of Montreal's Faculty of Medicine [13].

In addition, there were few \#icanhazpdf requests for articles from journals in the subject categories of pharmacology

Table 3. Requestor's employment sector.

\begin{tabular}{lcr}
\hline & $\begin{array}{c}\text { Number } \\
\text { of tweets }\end{array}$ & $\begin{array}{r}\text { Percent } \\
\text { total }\end{array}$ \\
\hline Academic (non-librarian) & 85 & $28.1 \%$ \\
Student & 41 & $13.6 \%$ \\
Journalist/writer/public relations & 19 & $6.3 \%$ \\
Librarian & 10 & $3.3 \%$ \\
Clinician & 5 & $1.7 \%$ \\
Industry & 5 & $1.7 \%$ \\
Nonprofit, patient organization & 5 & $1.7 \%$ \\
Nonprofit, other & 4 & $1.3 \%$ \\
Independent consultant & 4 & $1.3 \%$ \\
Patient & 3 & $1.0 \%$ \\
Government & 3 & $1.0 \%$ \\
Teacher (non-post-secondary) & 1 & $0.3 \%$ \\
Unknown & 117 & $38.7 \%$ \\
Total & 302 & $100.0 \%$ \\
\hline
\end{tabular}


( $n=18)$, nursing $(n=14)$, clinical psychology $(n=2)$, and the health professions $(n=5)$. These results may again be related to rates of Twitter adoption among these professional groups. Studies report Twitter usage rates of less than 10\% among pharmacy preceptors [14], undergraduate pharmacy students [15], and new graduate nurses [16].

Although originally envisioned for users without institutional library access, \#icanhazpdf requests from user groups such as journalists, patient organizations, and patients were limited $(n=27)$. During the study period, $45 \%$ of requests $(n=136)$ were from Twitter users affiliated with a postsecondary academic institution such as faculty members, students, and librarians. If tweets from requestors with unknown affiliation are excluded, over $70 \%$ of requestors were affiliated with a post-secondary academic institution $(n=136 / 185)$. These results are consistent with earlier research by Liu [10]; the results of her 2013 study indicated that users from occupational groups with institutional library access made a majority of \#icanhazpdf requests.

Faculty and student use of \#icanhazpdf warrants further analysis in light of academic users' considerable access to library resources and services such as interlibrary loan. Although a full investigation of user motivations is not within the scope of this study, comments tweeted by \#icanhazpdf users during the study period can provide some insights into this particular article sharing practice.

Evidence from a study conducted by Connaway et al. [17] suggests that convenience, including ease of use and speed, is "one of the primary criteria used for making choices during the information-seeking process". \#icanhazpdf is fast, and users may retrieve the requested article within minutes. Several tweets compared the speed of \#icanhazpdf to the speed of interlibrary loan services:

Using \#icanhazpdf has been so helpful and fast (only used a few times). Way faster than ILL requests

Trying this b/c ILL is slow for what should be an EZ request

Still haven't gotten paper 22 minutes after \#icanhazpdf request. The Internet is so over.

\#icanhazpdf is also relatively easy and convenient. Anyone with a Twitter account can use the hashtag to obtain articles quickly and without much effort. \#icanhazpdf users do not need to remember (or even have) library login credentials. These sample tweets suggest that the convenience of \#icanhazpdf is an important consideration for users:

Can anyone help out with this oldie and save me a trip to the library?

Working from home ... can anyone help - Journal of Medicinal Chemistry

Dear \#lazyweb, \#icanhazpdf - pretty please?

Opportunities to connect with other researchers may serve as an additional motivating factor. In an ethnographic study, Veletsianos [18] observes that social media is "a place where scholars can congregate to share their work, ideas and experiences ... Through social media gatherings, distributed individuals build ties, bonds and solidarity, even when they may have not met each other face-to-face". A small number of \#icanhazpdf requests included in the current study $(n=9)$ were direct replies to article links shared by other researchers. In such cases, an \#icanhazpdf request may indicate shared interests around a particular article or topic, and may also foster connections between researchers. Sample tweets include:

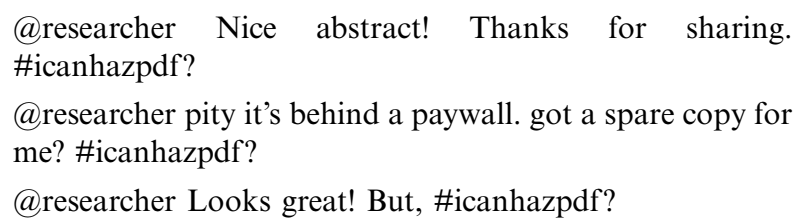

In addition, use of the \#icanhazpdf hashtag may also serve as a form of social protest and a sign of discontent with current academic publishing practices and economic systems. Dunn et al. [19] and Kroll [20] position peer-topeer article sharing as an act of civil disobedience, and \#icanhazpdf creator Andrea Kuszewski has recently stated that use of the hashtag is "not an aggressive act but it's just a way of saying things need to change" [21]. Veletsianos argues that article sharing among researchers exemplifies academic researcher values surrounding sharing and openness [18].

\section{Limitations}

A primary limitation of this study is its reliance on a convenience sample; results are not generalizable. The study sample is also potentially incomplete, as RowFeeder software cannot guarantee instantaneous tweet capture. In addition, the study relies on self-reported Twitter profile information, which may not be accurate. A further potential limitation is subjectivity in the authors' coding of tweets. While categorizing \#icanhazpdf tweets according to characteristics such as geographic region and journal subject area was straightforward, classifying requestors' employment sector was somewhat subjective due to differences in academic ranks in North America, the United Kingdom, and Europe, as well as ambiguity in the information provided in requestors' Twitter profiles.

Although this study presents preliminary observations of \#icanhazpdf user motivations as extrapolated from users' comments on Twitter, further research and analysis are recommended in this area. Although not within the scope of the present study, the copyright and licensing implications surrounding scholarly article sharing are another important area for further research and discussion.

\section{Conclusion}

The current study identified 302 requests for health sciences literature using the \#icanhazpdf Twitter hashtag during a three-month period. The majority of requests were for articles from medical journals. The number of requests is not large, but health sciences librarians should be aware of \#icanhazpdf as yet one more avenue of scholarly sharing.

Nearly half of the requests were made by individuals whose Twitter profiles indicated some affiliation with a 
post-secondary academic institution. Such requests are evidence of users choosing social media over the library as a means of obtaining scholarly materials; librarians can and should examine the use of \#icanhazpdf for the insights it provides into user behaviour.

As tweets in the current study suggest, individuals affiliated with post-secondary institutions may turn to Twitter to obtain health sciences literature for a variety of reasons. Researchers may appreciate the peer-to-peer connections fostered through \#icanhazpdf requests, or may use this article retrieval method to signal discontent with current academic publishing models. \#icanhazpdf users may also turn to Twitter for reasons relating to convenience, ease of use, speed, or even lack of knowledge of library services and processes. The \#icanhazpdf phenomenon is thus a rich source of information for health sciences librarians - and indeed all academic librarians - who are seeking to understand patron behaviour and improve library services in the areas of scholarly communication, library instruction, and interlibrary loan.

\section{References}

1. Kurata K, Morioka T, Yokoi K, Matsubayashi M. Remarkable growth of open access in the biomedical field: analysis of PubMed articles from 2006 to 2010. PLoS One. 2013;8(5): e60925. doi:10.1371/journal.pone.0060925.

2. Salo D. Academic Samizdat [Internet]. [place unknown]: ScienceBlogs; 2010 [cited 30 Nov 2015]. Available from: http://scienceblogs.com/bookoftrogool/2010/02/09/academicsamizdat/

3. Wren JD. Open access and openly accessible: a study of scientific publications shared via the internet. BMJ. 2005; 330(7500):1128. doi:10.1136/bmj.38422.611736.E0.

4. Masters K. Opening the non-open access medical journals: Internet-based sharing of journal articles on a medical website. Internet $J$ Med Informat [Internet]. 2008 [cited 30 Nov 2015];5(1):1-9. Available from: http://ispub.com/IJMI/5/1/6971

5. Cabanac G. Bibliogifts in LibGen? A study of a text-sharing platform driven by biblioleaks and crowdsourcing. $J$ Assoc Informat Sci Technol. 2015. doi:10.1002/asi.23445.

6. Greenhill K, Wiebrands C. No library required: the free and easy backwaters of online content sharing. In: VALA 2012: 16th Biennial Conference and Exhibition [Internet]; 2012 Feb 6-9; Melbourne, Australia. [cited 30 Nov 2015]. Available from: http://espace.library.curtin.edu. $\mathrm{au} / \mathrm{R}$ ?func $=$ dbin-jump-full\&local_base=gen01-era02\&object_ $\mathrm{id}=180950$

7. England M, Jones P. Diversification of access pathways and the role of demand-driven acquisition: a case study at the University of Utah. Serials Libr. 2014;66(1-4):96-105. doi:10.1080/0361526X.2014.879012.

8. Clarke, C. The end of an era for Academia.edu and other academic networks? [Internet]. Wheat Ridge, CO: The Scholarly Kitchen; 2013 [cited 22 Jan 2016]. Available from: http://scholarlykitchen.sspnet.org/2013/12/11/has-elseviersignaled-a-new-era-for-academia-edu-and-other-professionalnetworks/

9. Gardner CC, Gardner GJ. Bypassing interlibrary loan via twitter: an exploration of \#icanhazpdf requests. In: ACRL 2015 [Internet]; 2015 Mar 25-28; Portland (OR). [cited 30 Nov 2015]. Available from: http://www.ala.org/acrl/sites/ala. org.acrl/files/content/conferences/confsandpreconfs/2015/ Gardner.pdf

10. Liu J. Interactions: the numbers behind \#ICanHazPDF [Internet]. London: Altmetric.com; 2013 [cited 30 Nov 2015]. Available from: http://www.altmetric.com/blog/interactionsthe-numbers-behind-icanhazpdf/

11. RowFeeder [Internet]. Seattle (WA): Simply Measured; 2011 [cited 22 Jan 2016]. Available from: https://rowfeeder.com/

12. Rich P. Where is everyone? Canadian physician use of social media. In: Medicine 2.0 Summit \& World Conference [Internet]; 2014 Nov 13-14; Maui, Hawaii. [cited 30 Nov 2015]. Available from: http://www.medicine20congress.com/ ocs/index.php/med/med2014/paper/view/2202

13. Clairoux N, Weiss-Lambrou R. Social media use in medical and health professional education: role of the librarian on a faculty steering committee. In: Canadian Health Libraries Association Conference [Internet]; 2015 Jun 19-22; Vancouver, Canada. [cited 30 Nov 2015]. Available from: http://hdl. handle.net/1866/12222

14. Kukreja P, Heck Sheehan A, Riggins J. Use of social media by pharmacy preceptors. Am J Pharm Educ. 2011;75(9):176. doi:10.5688/ajpe759176.

15. Clauson K, Singh-Franco D, Sircar-Ramsewak F, Joseph S, Sandars J. Social media use and educational preferences among first-year pharmacy students. Teach Learn Med. 2013;25:122-8. doi:10.1080/10401334.2013.770742.

16. Tuckett A, Turner C. Do you use social media? A study into new nursing and midwifery graduates' uptake of social media. Int J Nurs Pract. 2015. doi:10.1111/ijn.12411.

17. Connaway LS, Dickey TJ, Radford ML. "If it is too inconvenient I'm not going after it": convenience as a critical factor in information-seeking behaviors. Libr Inform Sci Res. 2011;33(3):179-90. doi:10.1016/j.lisr.2010.12.002.

18. Veletsianos G. Open practices and identity: evidence from researchers and educators' social media participation. $\mathrm{Br} J$ Educ Technol. 2013;44(4):639-51. doi:10.1111/bjet.12052.

19. Dunn AG, Coiera E, Mandl KD. Is biblioleaks inevitable? J Med Internet Res. 2014;16(4):e112. doi:10.2196/jmir.3331.

20. Kroll D. \#icanhazpdf: civil disobedience? [Internet]. Washington (DC): Terra Sigillata; 2011 [cited 30 Nov 2015]. Available from: http://cenblog.org/terra-sigillata/2011/12/22/ icanhazpdf-civil-disobedience/

21. Mohdin A. Academics have found a way to access insanely expensive research papers - for free [Internet]. Washington (DC): Quartz; 2015 [cited 30 Nov 2015]. Available from: http://qz.com/528526/academics-have-found-a-way-to-accessinsanely-expensive-research-papers-for-free/ 